\title{
Crowdsourcing visual perception experiments: A case of contrast threshold
}

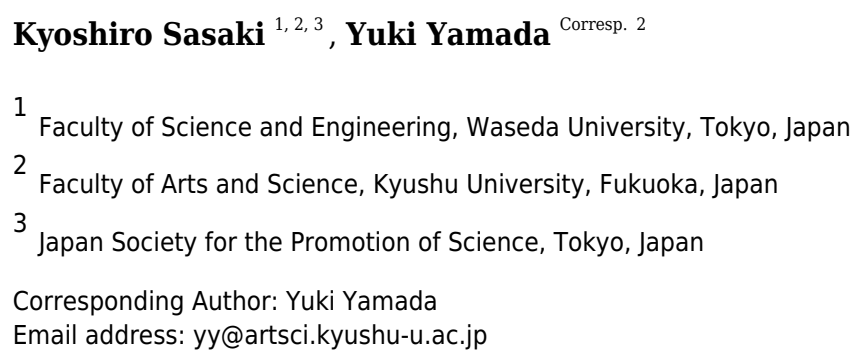

Crowdsourcing has commonly been used for psychological research but not for studies on sensory perception. A reason is that in online experiments, one cannot ensure that the rigorous settings required for the experimental environment are replicated. The present study examined the suitability of online experiments on basic visual perception, particularly the contrast threshold. We conducted similar visual experiments in the laboratory and online, employing three experimental conditions. The first was a laboratory experiment, where a small sample of participants ( $n=24$; laboratory condition) completed a task with 10 iterations. The other two conditions were online experiments: participants were either presented with a task without repetition of trials ( $n=285$; online nonrepetition condition) or one with 10 iterations ( $n=166$; online repetition condition). The results showed significant equivalence in the contrast thresholds between the laboratory and online repetition conditions, although a substantial amount of data needed to be excluded from the analyses in the latter condition. The contrast threshold was significantly higher in the online non-repetition condition compared with the laboratory and online repetition conditions. To make crowdsourcing more suitable for investigating the contrast threshold, ways to reduce data wastage need to be formulated. 
3 Crowdsourcing visual perception experiments: A case of contrast

4

5

6

7

8

9

10

11

12

13

$14{ }^{*}$ Correspondence:

15

Dr. Yuki Yamada

16

17744 Motooka, Nishi-ku, Fukuoka, 819-0395, Japan.

18 E-mail: yamadayuk@gmail.com

19

TEL \& FAX: +81-92-802-5837

\section{threshold}

Kyoshiro Sasaki ${ }^{1,2,3}$ and Yuki Yamada ${ }^{2 *}$

${ }^{1}$ Faculty of Science and Engineering, Waseda University, Tokyo, Japan

${ }^{2}$ Faculty of Arts and Science, Kyushu University, Fukuoka, Japan

${ }^{3}$ Japan Society for the Promotion of Science, Tokyo, Japan

Running head: Crowdsourcing meets Visual Perception

20

21 
24 Crowdsourcing has commonly been used for psychological research but not for studies on sensory 25 perception. A reason is that in online experiments, one cannot ensure that the rigorous settings 26 required for the experimental environment are replicated. The present study examined the 27 suitability of online experiments on basic visual perception, particularly the contrast threshold. We 28 conducted similar visual experiments in the laboratory and online, employing three experimental 29 conditions. The first was a laboratory experiment, where a small sample of participants $(n=24$; 30 laboratory condition) completed a task with 10 iterations. The other two conditions were online 31 experiments: participants were either presented with a task without repetition of trials $(n=285$; 32 online non-repetition condition) or one with 10 iterations ( $n=166$; online repetition condition).

33 The results showed significant equivalence in the contrast thresholds between the laboratory and 34 online repetition conditions, although a substantial amount of data needed to be excluded from the 35 analyses in the latter condition. The contrast threshold was significantly higher in the online nonrepetition condition compared with the laboratory and online repetition conditions. To make 37 crowdsourcing more suitable for investigating the contrast threshold, ways to reduce data wastage 38 need to be formulated.

40 Keywords: Online experiment, Perception, Vision, Contrast threshold 
Over the last decade, experiments in psychological research have gone beyond the

44 laboratory. The increasing diversity of research methods and technological advances has increased 45 opportunities for researchers to use resources outside the laboratory. For example, researchers are 46 using outsourcing services to recruit experimental participants and, often, even commissioning 47 research firms to conduct their surveys and experiments. In addition, based on outstanding 48 technological advances in the digital environment and mobile information devices, 49 "crowdsourcing," which is the practice of asking many unspecified people to various kinds of tasks via the internet, has become a powerful tool for psychological research (for a review, see Stewart, 51 Chandler, \& Paolacci, 2017).

52 Crowdsourcing can be used for data collection and in asking large numbers of people to participate in surveys or experiments via the internet. Service providers (e.g., Amazon and Yahoo!)

54 manage an experimenter's task and act as a payment agency. The use of crowdsourcing has a number of advantages. The first is its very low cost (e.g., Stewart et al., 2017); for example, participants receive less than 1 USD for responding to a simple questionnaire or engaging in an easy cognitive task. Second, large (more than 1,000 people) and diverse (in age, sex, and culture) samples can easily be employed. The ease in collecting large amounts of diverse data is beneficial not only from the perspective of random sampling but also for planning experiments and estimating the effect size prior to conducting the experiment (Chrabaszcz, Tidwell, \& Dougherty, 2017). Third, it enables researchers to use their time efficiently. With experiments running all hours of the day and night, data from 1,000 people can be obtained within a day or two, depending on how many active users are registered with the service. 
66 questionnaires (e.g., Crangle \& Kart, 2015; Garcia, Kappas, Küster, \& Schweitzer, 2016; Gottlieb

67 \& Lombrozo, 2018; Hurling et al., 2017; Sasaki, Ihaya, \& Yamada, 2017) and crowdsourced tasks:

68 visual search (de Leeuw \& Motz, 2015), reaction time (e.g., Nosek, Banaji, \& Greenwald, 2002;

69 Sasaki et al., 2017; Schubert, Murteira, Collins, \& Lopes, 2013), keystroke (Pinet et al., 2017),

70 Stroop (Barnhoorn, Haasnoot, Bocanegra, \& van Steenbergen, 2014;

71 Crump, McDonnell, \& Gureckis, 2013; Majima, 2017), attentional blink (Barnhoorn et al., 2014;

72 Brown et al., 2014), flanker (Simcox \& Fiez, 2014; Majima, 2017; Zwaan et al., 2018), Simon

73 (Majima, 2017; Zwaan et al., 2018), lexical decision (Simcox \& Fiez, 2014), category learning

74 (Crump et al., 2013), memory (Brown et al., 2014; Zwaan et al., 2018), priming (Zwaan et al.,

75 2018), and decision-making tasks (Berinsky, Huber, \& Lenz, 2012; Brown et al., 2014). A

76 previous study using auditory stimuli likewise employed crowdsourcing (Woods, Siegel, Traer, \&

77 McDermott, 2017). A recent study recruited infants aged five to eight months via crowdsourcing

78 and measured their looking time with webcams (Tran, Cabral, Patel, \& Cusack, 2017). These

79 studies have suggested that the effect size of the performance in such tasks is comparable to that

80 in laboratory experiments; hence, crowdsourcing can be used for diverse online experiments with

81 publishable reliability.

82 However, conventional studies on sensory perception are completed in the laboratory.

83 Moreover, only authors or their laboratory members, who should be well experienced with

84 psychophysical measurements, often participate in experiments on sensory perception. Only a

85 small number of studies have attempted to run sensory perceptual experiments via crowdsourcing.

86 Previous studies have investigated color (Lafer-Sousa, Hermann, \& Conway, 2015; Szafir, Stone,

87 \& Gleicher, 2014) and randomness (Yamada, 2015) on the web but used one-time color-matching,

88 color word selection, forced choices (same or different), or magnitude estimation tasks. A few

89 studies have measured the point of subjective equality, sensitivity, or thresholds using 
90 psychophysical methods in studies on color perception (Ware et al., 2018), volume perception

91 (Pechey et al., 2015), size perception (Brady \& Alvarez, 2011) scene perception (Brady, Shafer-

92 Skelton, \& Alvarez, 2017), and stimulus visibility (Bang, Shekhar, \& Rahnev, 2019). One reason

93 that experiments on sensory perception are rarely conducted online is the necessity for rigorous

94 control over the experimental environment. Online experiments depend significantly on the

95 participant's own computing environment, and experimenters cannot control the display settings,

96 visual distance (or visual field), or lighting conditions. Thus far, online experiments seem

97 unsuitable for experimental studies that focus on the visual functions of spatial and temporal

98 resolutions. For example, in examining the issue of the temporal aspect of stimulus presentation,

99 researchers have found that stimuli are systematically presented for $20 \mathrm{~ms}$ longer than the

100 programmed durations (de Leeuw \& Motz, 2015; Reimers \& Stewart, 2014). However, the above

101 concerns might be negligible, and crowdsourcing is possibly suitable for perception studies. In this

102 case, a large sample could be recruited to bring sufficient statistical power. Further, large and

103 diverse samples are beneficial for the examination of individual differences in perception studies.

104

Aim of the present study. This study focused on measuring low-level visual perception via

105

online experiments. We examined the contrast threshold in vision via online crowdsourcing and

106 laboratory experiments. Contrast threshold is a non-temporal visual capacity that is highly

107 susceptible to the influence of the display condition during measurement. Its measurement needs

108 strict linearization of the output of the display with gamma correction; however, most displays of

109 home PCs are not linearized. Moreover, the viewing distance should vary across the participants

110 in the online condition; the spatial frequency depends on this distance. We believed that a

111 comparison between web and lab measurements of visual contrast thresholds would provide

112 tangible evidence of what online experiments can and cannot test regarding non-temporal aspects

113 of stimulus presentation. If the non-linearity of monitor displays, differences in the viewing 
114 distance, and other possible factors comprise a negligible random effect, then the contrast threshold

115 online and in the laboratory would be similar. Another important issue is boredom in the

116 participants. In online experiments, boredom in participants substantially decreases data quality

117 (Chandler, Mueller, \& Paolacci, 2014); many repetitions are likely to induce boredom. Thus, the

118 present study used two types of iteration for online experiments: the repetition and non-repetition

119 conditions. In the former, participants were presented with each trial 10 times per stimulus

120 condition, whereas in the latter condition, each trial was presented only once. If we could control

121 for measurement errors or individual differences by increasing the sample size, then a single trial

122 for a stimulus condition would suffice to lead to an appropriate conclusion, even in online

123 experiments, without data deterioration. For this reason, the sample size of the participants in the

124 non-repetitive condition was about 10 times that of the repetitive condition.

125

126

\section{Methods}

127 Participants. We used G*Power to determine the sample sizes needed for the repetition condition $128(\alpha=.05,1-\beta=.80)$. In the laboratory condition, we used a moderate effect size $(f=.25)$ in the 129 calculation of the required sample size. The required and maximum sample size was 24 . In the 130 online repetition condition, we used a small effect size $(f=.10)$ in the calculation of the required 131 sample size, because of the potential for noise in the data from online experiments. The required 132 sample size was revealed to be 138. Considering potential satisficers (Chandler et al., 2014; 133 Oppenheimer, Meyvis, \& Davidenko, 2009), who do not devote an appropriate amount of 134 attentional resources to a task and hence cursorily perform it, 200 people was set as the maximum 135 sample size; participants were recruited through a crowdsourcing service (Yahoo! Crowdsourcing: 136 http://crowdsourcing.yahoo.co.jp/). The required sample size in the online non-repetition 137 condition was at least 10 times that in the laboratory condition ( 240 people) according to the 
138 differences in the number of repetitions. Similarly, in the online repetition condition, we recruited

139300 people as the maximum sample size to account for the potential influence of satisficers. The

140 participants in the laboratory conditions undertook several experiments, including the present

141 experiment, for three hours, and subsequently received 4,000 JPY (the present experiment itself

142 took less than $30 \mathrm{~min}$, although we did not accurately record the duration). The order of these

143 experiments was randomized across the participants. The participants in the online repetition and

144 non-repetition conditions received 50 and $20 \mathrm{~T}$-points (Japanese point service, in which $1 \mathrm{~T}$-point

145 is worth $1 \mathrm{JPY})^{1}$, respectively. The participants were not made aware of the purpose of the study.

146 The experiment was conducted according to the principles laid down in the Helsinki Declaration.

147 The protocol was approved by the ethics committees of Waseda University (approval number:

148 2015-033) and Kyushu University (approval number: 2016-017). We obtained written informed

149 consent from all of the participants in the laboratory condition. Meanwhile, it was difficult to

150 obtain written informed consent in the online conditions. Thus, according to the protocol (approval

151 number: 2016-017), we explained the details of the online experiments in instructions sections,

152 and then asked the participants to take part in the experiments only when they agreed to the

153 instructions. We recruited only PC users to participate in the online experiment.

154 Apparatus. In the laboratory condition, stimuli were presented on a 23.5 -inch LCD display 155 (FG2421; EIZO, Japan). The resolution of the display was $1920 \times 1080$ pixels, and the refresh rate 156 was $100 \mathrm{~Hz}$. We performed gamma correction for the luminance emitted from the monitor. The 157 presentation of stimuli and the collection of data were computer-controlled (Mac mini, Apple, 158 USA). We used MATLAB with the Psychtoolbox extension (Brainard, 1997; Pelli, 1997) to 159 generate the stimuli. The observer's visual field was fixed using a chin-and-head rest at a viewing 160 distance of $57 \mathrm{~cm}$ from the display. The size information at the visual angle described for the 161 laboratory condition was based on this viewing distance. In the online conditions, the experiment 
162 was conducted on a web browser with a JavaScript application (jsPsych; de Leeuw, 2015). jsPsych

163 is a useful toolbox for psychological research, employed in several previous studies (de Leeuw \&

164 Motz, 2016; Pinet et al., 2017; Sasaki et al., 2017).

165 Stimuli and procedure. Stimuli consisted of a fixation circle (diameter of 0.24 degrees) and Gabor 166 patches, the diameter of which was 42 pixels (2 degrees in the laboratory conditions). The SD of 167 a gaussian function was 6 pixels $\left(0.29^{\circ}\right)$. There were four spatial frequencies of the carrier: 0.02 , $1680.05,0.09$, and 0.38 cycles per pixel (cpp; $0.5,1,2$, and 8 cycles per degree [cpd] in the laboratory 169 conditions). We set seven contrast levels (the Michelson contrast), varying across the spatial 170 frequencies. The contrasts in the $0.02 \mathrm{cpp}(0.5 \mathrm{cpd})$ trials were $3 \%, 8 \%, 13 \%, 18 \%, 23 \%, 28 \%$, 171 and $33 \%$. The contrasts in the 0.05 and $0.09 \mathrm{cpp}$ ( 1 and $2 \mathrm{cpd}$ ) trials were $1 \%, 6 \%, 11 \%, 16 \%$, $17221 \%, 26 \%$, and $31 \%$. The contrasts in the $0.38 \mathrm{cpp}(8 \mathrm{cpd})$ trials were $5 \%, 10 \%, 15 \%, 20 \%, 25 \%$, $17330 \%$, and $35 \%$. The Gabor patches were tilted $45^{\circ}$ clockwise or counterclockwise. We took 174 screenshots of the stimuli on the monitor at the laboratory and then used them for the online 175 conditions.

176 In the laboratory condition, the experiment was conducted in a darkened room. Figure 1 177 shows the timeline of a trial in each of the conditions. The participants initiated each trial by 178 pressing the space key. The fixation circle was presented for $500 \mathrm{~ms}$. After the fixation circle 179 disappeared, the Gabor patch was presented for $50 \mathrm{~ms}$. Then, a blank screen was presented for 300 180 ms, followed by the prompt: "In which direction was the stimulus tilted?" The participants were 181 asked whether the stimulus was tilted clockwise or counterclockwise. They responded without 182 time limits or feedback. Each of the spatial frequency conditions was conducted in a separate 183 session; thus, the experiment consisted of four sessions. The session order was randomized across 184 the participants. In each session, trials were conducted for seven contrasts in two orientations. In 185 the repetition condition, each combination of contrast and orientation was presented 10 times per 
186

187

188

189

190

191

192

193

194

195

196

197

198

199

200

201

202

203

204

205

206

207

208

209

session. Thus, participants in the repetition condition completed 560 trials, whereas those in the non-repetition condition completed only 56 . The order of the trials was also randomized across the participants. Before the first session, we conducted a practice session, in which the participants completed four trials. The spatial frequency of the practice session was identical to that of the first session, and the contrast was $100 \%$. Both of the orientations appeared twice. As in the experiment conditions, the trial order of each session was randomized across the participants.

In the online conditions, the participants accessed the page of Crowdsourcing for the link to the web address of the experiment. They navigated to the experiment page via the web address and then input their age and sex. Moreover, after completing the experiment, a four-digit number (8382 and 3599 in the online repetition and non-repetition conditions, respectively) was presented at the final experiment page; the participants typed this number on an empty form on the Yahoo! Crowdsourcing page. The four-digit number was registered for Yahoo! Crowdsourcing in advance. Only when the input and registered numbers corresponded would Yahoo! Crowdsourcing acknowledge that the participants had completed the experiment and give the reward. If the input and registered numbers did not correspond, Yahoo! Crowdsourcing made the participants drop out and did not give the reward. The procedures were identical to that of the laboratory conditions, except for the added insertion of attention check questions (ACQs). This additional step was included because online participants are often distracted (Chandler et al., 2014) or are satisficers (Oppenheimer et al., 2009). ACQs can reduce low-quality responses (Aust, Diedenhofen, Ullrich, \& Musch, 2012; Oppenheimer et al., 2009). These tend to be easy calculations based on the four basic arithmetic operations (e.g., $20+15=$ ?). In the present study, ACQs appeared halfway through the total number of trials in each session and participants selected the correct answer from five options. We conducted the online repetition and online non-repetition conditions from January 25 to 28,2019 and January 29 to February 7, 2019, respectively. 
210 Data analysis. We excluded participants who gave incorrect answers to one or more of the ACQs.

211 In the laboratory and online repetition conditions, we calculated the contrast threshold of each

212 spatial frequency for each participant, for which the proportion of "correct" responses was 0.82

213 (Cameron, Tai, \& Carrasco, 2002; Lee, Baek, Lu, \& Mather, 2014), using a probit analysis (i.e.,

214 fitting a cumulative Gaussian function to the proportion of "correct" responses as a function of the

215 contrast level). We used the "glm" function in $\mathrm{R}$ (3.4.4). The probit analysis provided the means

216 and standard deviations (SDs) of the distributions. Then, we calculated the contrast thresholds

217 using the means, SDs, and the "qnorm" function in R. We excluded participant data when $\beta$

218 calculated by the probit analysis was a negative value. This negative value indicated a reduction

219 in correct responses as the contrast level increased. In such cases, we could not calculate the

220 thresholds. We also excluded the data from participants whose contrast thresholds were less than

2210 or greater than $100 \%$ because the contrast threshold should be within this range. In the online

222 non-repetition condition, we used the pooled data from all the participants and then calculated the

223 contrast threshold for each spatial frequency by the same procedure of the repetition condition.

224 First, to confirm whether the contrast threshold depended on the spatial frequency, we

225 conducted a one-way analysis of variance (ANOVA) on the contrast thresholds, with spatial

226 frequency as a within-participant factor, for the laboratory and online repetition conditions. We set

227 the alpha level at .05 and calculated $\eta_{p}{ }^{2}$. When the main effects were significant, we conducted

228 multiple comparison tests using Holm's method (Holm, 1979). We conducted the $t$-test six times.

229 Therefore, we increased $\alpha$ from .008 to .05 based on Holm's correction (Holm, 1979).

230

As our purpose was to examine whether the contrast thresholds were different or equivalent

231 between experimental environments in each spatial frequency, we conducted two-tailed Welch's

$232 t$-tests for the contrast thresholds for each spatial frequency. After the $t$-tests, we conducted 233 equivalence tests for the pairs in which the contrast thresholds were not significantly different. For 
234 the equivalence tests, we used the TOSTER package in R (Lakens, Scheel, \& Isager, 2018) and

235 set Cohen's $d$ to 0.5 . We compared the contrast threshold of the laboratory condition and the online

236 repetition and non-repetition conditions; thus, we had to conduct $t$-tests and equivalence test three

237 times at most. Therefore, we set $\alpha$ from .017 to .05 based on Holm's correction (Holm, 1979).

238

239

\section{Results}

240 The results of the proportion of the correct responses and the thresholds in the laboratory and

241 online experiments are shown in Figures 2 and 3, respectively. We collected data from 24 people

242 in the laboratory condition. In the online repetition condition, of the 200 people recruited, only 80

243 participated ${ }^{1}$. As this number did not reach the required sample size, we recruited another 200

244 people and 86 people participated. Hence, we collected data from 166 people in total. For the

245 online non-repetition condition, of the 300 people recruited, only 156 participated. Therefore, we

246 recruited another 250 people and 129 people participated. Hence, we collected data from 285

247 people in total. We excluded the data from 2 (one owing to a negative $\beta$ and the other, for having

248 a contrast threshold greater than $100 \%), 84$ (53 owing to a negative $\beta ; 13$, a contrast threshold less

249 than 0 ; 8, a contrast threshold greater than 100\%; and 10, wrong answers to ACQ), and 19 (all

250 owing to wrong answers to ACQ) participants in the laboratory, online repetition, and online non-

251 repetition conditions, respectively, based on the rules detailed in the Data analysis section. Thus,

252 we submitted the data from 22 (16 males and 6 females, mean age $\pm \mathrm{SEM}=21.39 \pm 0.39$ ), 82 (54

253 males, 26 females, and 2 non-respondents, mean age $\pm \mathrm{SEM}=43.56 \pm 1.04)$, and $266(176$ males

254 and 90 females, mean age $\pm \mathrm{SEM}=42.92 \pm 0.61$ ) participants in the laboratory, online repetition,

255 and online non-repetition conditions, respectively, for the statistical analyses.

256 Effects of spatial frequency within the laboratory and online repetition conditions. The results of 257 the ANOVA on the contrast thresholds in the laboratory condition revealed that the main effect 
258 was significant, $F(3,63)=7.63, p<.001, \eta_{p}^{2}=.27$. The multiple comparison tests showed that the

259 threshold was significantly higher in the $0.5 \mathrm{cpd}$ trials compared with the 1 and $2 \mathrm{cpd}$ trials, $t \mathrm{~s}(21)$

$260>6.25, p \mathrm{~s}<.001$, Cohen's $d \mathrm{zs}>1.33$. Moreover, the threshold was significantly higher in the $4 \mathrm{cpd}$

261 trials compared with the 2 cpd trials, $t(21)=2.88, p=.009$, Cohen's $d z=0.61$. The results of the

262 ANOVA on the contrast thresholds in the online repetition condition revealed that the main effect

263 was significant, $F(3,243)=26.23, p<.001, \eta_{p}{ }^{2}=.24$. The multiple comparison tests showed that

264 the threshold was significantly higher in the 4 cpd trials compared with the 1 and 2 cpd trials, $265 t \mathrm{~s}(81)>6.77, p \mathrm{~s}<.001$, Cohen's $d \mathrm{zs}>0.74$. The threshold was also significantly higher in the 0.5

266 cpd trials compared with the 1 and 2 cpd trials, $t \mathrm{~s}(81)>4.98, p \mathrm{~s}<.001$, Cohen's $d \mathrm{zs}>0.64$.

267 Moreover, we calculated a McFadden's pseudo $R^{2}$ for each of spatial frequency in the laboratory

268 and online repetition conditions and performed the two-way ANOVA on McFadden's pseudo $R^{2}$

269 with spatial frequency as a within-participant factor and experimental circumstances as a between-

270 participant factor ${ }^{2}$. As a result, the main effect of spatial frequency was significant $(F(3,306)=$

$\left.27127.88, p<.001, \eta_{p}^{2}=.21\right)$. Importantly, the main effect of experimental circumstances and

272 interaction were not significant (experimental circumstances: $F(1,102)=0.83, p=.37, \eta_{p}{ }^{2}=.008$;

273 interaction: $\left.F(3,306)=0.52, p=.67, \eta_{p}^{2}=.005\right)$.

274 Differences and equivalences between laboratory and repeated and non-repeated online

275 conditions. Table 1 shows the summary of the results. For the $0.5 \mathrm{cpd}$ trials, the threshold was

276 significantly higher in the online non-repetition condition compared with the online repetition,

$277 t(332.97)=6.14, p<.001$, Cohen's $d=0.51$, and laboratory conditions, $t(159.41)=5.95, p<.001$,

278 Cohen's $d=0.45$. Meanwhile, the online repetition and laboratory conditions showed no

279 significant difference, $t(68.92)=0.31, p=.76$, Cohen's $d=0.05$. The equivalence test showed 280 significant equivalence between the online repetition and laboratory conditions, $t(68.92)=2.26, p$ $281=.013$. 

condition compared with the online repetition, $t(314.58)=7.54, p<.001$, Cohen's $d=0.55$, and Cohen's $d=0.09$. The equivalence test showed significant equivalence between the online repetition and laboratory conditions, $t(82.43)=2.13, p=.018$.

For the 2 cpd trials, the threshold was significantly higher in the online non-repetition condition than in the online repetition, $t(319.24)=7.06, p<.001$, Cohen's $d=0.52$, and laboratory conditions, $t(268.92)=7.11, p<.001$, Cohen's $d=0.72$; no significant difference was found between the online repetition and laboratory conditions, $t(57.31)=0.33, p=.742$, Cohen's $d=$ laboratory conditions, $t(57.31)=2.12, p=.019$.

For the 4 cpd trials, the threshold was significantly higher in the online non-repetition condition compared with the online repetition, $t(344.97)=6.23, p<.001$, Cohen's $d=0.50$, and laboratory conditions, $t(56.41)=5.06, p<.001$, Cohen's $d=0.51$. However, no significant difference was found between the online repetition and laboratory conditions, $t(31.40)=0.56, p=$ .577 , Cohen's $d=0.14$. The equivalence test showed that the equivalence between the online repetition and laboratory conditions was marginally significant, $t(31.40)=1.48, p=.075$.

\section{Discussion}

302

This study examined whether the contrast threshold was properly measured in an online experiment with two conditions: a condition with repetition of trials and another without repetition. The results showed equivalences in the contrast thresholds of the online repetition and laboratory 
306 the online repetition and laboratory conditions. Thus, online experiments seem to be able to 307 measure the contrast threshold as adequately as laboratory experiments, provided enough 308 repetition $^{3}$. Notably, it is difficult to measure contrast thresholds without repetitions. However, as 309 discussed below, there was a high rate of exclusions. In this case, it might be difficult to obtain 310 large and diverse data; thus, one of the advantages of crowdsourcing is possibly lost. Taken 311 together, rash decisions to use crowdsourcing for perception studies is likely to be risky at this 312 time.

313 The present study excluded $51 \%$ of the data in the online repetition condition. These 314 exclusions mainly stemmed from the fact that the correct responses decreased as the contrast level 315 increased or the thresholds were under zero. That is, in the online repetition condition, the contrast 316 threshold could be barely calculated precisely. One possibility is that the experimental 317 environment of $49 \%$ of the participants in the online repetition condition might be similar to that 318 of the laboratory condition. We were able to calculate the thresholds of these participants and 319 found significant equivalences between the laboratory and online repetition conditions. 320 Meanwhile, the contrast thresholds were much higher in the online non-repetition condition. 321 Although it is difficult to interpret this result, one can argue that the repetitive performance of the 322 experimental task in the online repetition condition caused perceptual learning. It has been well 323 known that contrast discrimination increases with repeated practice or training (e.g., Sowden, 324 Rose, \& Davies, 2002; Yu, Klein, \& Levi, 2004). However, there are only 10 repetitions for each 325 stimulus in the online repetitive condition, and this little practice does not seem to cause sufficient 326 perceptual learning. Alternatively, the difference in the results with and without repetition may 327 provide clues for problems specific to online experiments. A large amount of the data was excluded 328 in the online repetition condition. Based on this, we can expect the data obtained via online 329 experiments to be noisy. Such noisy data might be included in and mediate the results of the online 
330 non-repetition condition. Given the large amount of data exclusion in the online repetition

331 condition and the results of the online non-repetition condition, we could not conclude that online

332 experiments are adequate for measuring the contrast threshold. Indeed, the contrast threshold

333 would be difficult to measure via crowdsourcing unless the lighting conditions of each online

334 participant can be measured and calibrated via camera.

There may be solutions for improving the situation of online measurements of the contrast

336 threshold. One would be to control the experimental environment of each participant in the online

337 experiments to match that of a laboratory experiment. A previous study proposed beneficial tips

338 for controlling the size of stimuli, distance from the monitor, sound volume, and brightness

339 (Woods, Velasco, Levitan, Wan, \& Spence, 2015). Woods et al. (2015) also provided a possible

340 way to adjust color, which seems to be difficult to control across online participants. They

341 referenced the hints from a psychophysical study (To, Woods, Goldstein, \& Peli, 2013) that

342 demonstrated that humans have the ability comparable to a photometer when asked to match two

343 patches in terms of brightness. The potential solution of Woods et al. (2015) was to ask participants

344 to video record their computer screen and a colorful object (reference object) close to the screen

345 using the camera on a mobile device, and then manually calibrate the screen color to the reference

346 object. At this time, these methods require much effort from the participants and experimenters,

347 and prone to technological difficulties; thus, they might not be ultimately effective. The ways to

348 control experimental environments easily should lead to a reduction in low-quality data, and to a

349 decrease in the exclusion of data, while also maintaining the ease of online experiments via 350 crowdsourcing.

Another solution is related to participant negligence. In the online experiment, participants

352 might have a difficulty maintaining their motivation while performing tasks; for instance, they

353 may have been unprepared to participate in a psychological experiment and not met the 
354 experimenters. Participants with inconsistent motivation often do not devote enough effort to the 355 tasks, and, hence, cursory responses increase (satisficing, e.g., Berinsky, Margolis, \& Sances, 356 2016; Maniaci \& Rogge, 2014; Miura \& Kobayashi, 2016; Oppenheimer et al., 2009). ACQs, 357 which we set during the online condition sessions, are beneficial for protecting the quality of the 358 data from satisficing. It is easy for participants to answer ACQs correctly when they perform the 359 tasks carefully. Generally, it is important to exclude the data from those who wrongly answer 360 ACQs because of inattention and/or cursory responses, to improve the quality of data. However, 361 in the present study, the data exclusion owing to incorrect ACQ responses accounted for $6 \%$ of the 362 total data in each of the online conditions. Thus, the ACQ might not have worked as intended in the present study. The type of ACQ was extremely different from that of the main task (i.e.,judging the orientation of the Gabor patch). Given this, the ACQ could be improved so that participants 365 are not easily caught out, or another method could be used. An instructional manipulation check (IMC) is also helpful for detecting satisficers (Oppenheimer et al., 2009). An IMC checks whether

367 368 369 370 371 372 373 374 375 376 377 the participants carefully read the instructions for the tasks. Specifically, they can incorporate the instruction not to answer questions into some methods commonly used in psychological research (e.g., Likert scales); thus, if the participants do not carefully read the instructions, they mistakenly answer the questions. The data from such participants should be excluded because they improperly dealt with the tasks. Additionally, in a recent study, alerting satisficers to their inattentiveness by a repeated IMC was helpful in improving their information processing (Miura \& Kobayashi, 2016). In general, ACQs and IMCs are valid tools for the detection and exclusion of data from satisficers. However, it is difficult to prevent satisficers from participating in experiments. To avoid losing data owing to satisficers, blacklisting them might be more effective in the long term.

Other ways could be employed to maintain the quality of psychophysical online data. One is developing a platform designed for scientific research. Crowdsourcing services, such as Yahoo!

Peer) reviewing PDF | (2019:09:40944:3:0:NEW 21 Nov 2019) 
378 Crowdsourcing and Amazon Mechanical Turk, have some advantages for conducting

379 psychological research. However, they were not developed as research tools and have some

380 inconveniences as well. Recently, a platform for scientific research was designed (TurkPrime,

381 recently rebranded as CloudResearch: Litman, Robinson, \& Abberbock, 2017) and integrated with

382 Amazon Mechanical Turk. Prolific is also a remarkable platform for conducting surveys and

383 experiments online (Palan \& Schitter, 2018). These helpful systems for improving the quality of

384 online data have also been proposed: Excluding participants based on previous participation,

385 communicating with participants, and monitoring dropout and engagement rates. Elevating these

386 platforms should be helpful for improving the quality of data in online experiments.

387

Contrast sensitivity seemed to be lower in the present study than in the previous ones (e.g.,

388 Cameron et al., 2007; Lee et al., 2014). This discrepancy might be attributed to the intensity level

389 of the stimulus. Several studies have pointed out that the typical hardware used in psychological

390 studies (256 intensity levels, 8 bits) is insufficient for measuring contrast thresholds. One of the

391 solutions is to use a graphics card able to display more than 256 different luminance intensities

392 (e.g., Allard \& Faubert, 2008; Lu \& Dosher, 2013), but this does not seem to be realistic in online

393 experiments. A previous study proposed the solution of adding visual noise to the stimulus, thereby

394 not requiring special hardware (Allard \& Faubert, 2008). This solution might fit the context of

395 online experiments. We aim to address these issues in future studies.

396

Although crowdsourcing does not seem to be suitable for measurements of perception

397 studies at this time, the improvement of environments in online experiments will bring advantages.

398 For example, crowdsourcing enables researchers to obtain large amounts of data from various

399 people, which is advantageous for examining individual differences in perceptual and cognitive

400 processing. In classic laboratory experiments, most participants are university or graduate students,

401 and large amounts of data tend to be difficult to collect. The demographics, personal traits, and 
402 cognitive characteristics of the participants do not vary enough to examine the relation between

403 individual differences in perceptual and cognitive processing. Thus, this relation and underlying

404 mechanism have not been understood well, warranting further investigations (Yamada, 2015).

405 Crowdsourcing, however, allows researchers to recruit participants from around the world, and

406 hence, mass data from participants with various personality traits can be collected. Indeed, we and

407 others have already shown the relation between individual differences in personality traits (e.g.,

408 social anxiety, behavioral activation/inhibition systems, and mood) and emotional reactions using

409 crowdsourcing (Chaya et al., 2015; Sasaki et al., 2017). Moreover, we previously conducted a

410 perceptual study indicating the age and sex differences in the perception of pattern randomness

411 (Yamada, 2015). If the environment in online experiments is improved and crowdsourcing

412 becomes suitable for investigating visual perception, then online experiments will be helpful for

413 addressing issues regarding individual differences in visual perception.

414

415

Conclusions

416

The present study examined the suitability of online experiments on the contrast threshold.

417 As a result, online experiments seem to be able to measure the contrast threshold as adequately as

418 laboratory experiments, provided enough repetitions. However, there was a high rate of exclusions,

419 which is likely to spoil one of the advantages of crowdsourcing research. Thus, rash decisions to

420 use crowdsourcing for perception studies might be risky at this time. The improvement of

421 technology environments in online experiments via crowdsourcing will bring advantages;

422 individual differences in perceptual processing will be measurable. 
$425{ }^{1}$ Discrepancies between the number of recruitments and that of the actual participants were often

426 found when we used Yahoo! Crowdsourcing. We could not determine the exact reason. One

427 possibility was that the four-digit number was shared online (e.g., SNS) and some crowdworkers

428 may have seen it. In this case, they could be illegally admitted as completing the task by Yahoo!

429 Crowdsourcing even if they did not actually complete the task. Moreover, Yahoo! Crowdsourcing

430 allowed crowdworkers to access the recruiting page only once. Yahoo! Crowdsourcing manages

431 the number of those accessing the recruiting page via Yahoo ID. Crowdworkers, who had multiple

432 Yahoo IDs, could access the recruiting page several times. Therefore, after a participant had

433 completed our experiment, received the four-digit number, and taken the reward, they could access

434 the recruiting page with their other IDs again and input the four-digit number without performing

435 the experiment. These ways of hacking might have caused the discrepancy. Setting and generating

436 unique four-digit number for each participant could prevent this discrepancy; this is impossible at

437 the present system. We plan to discuss means for preventing these issues with Yahoo!

438 Crowdsourcing.

$439{ }^{2}$ We added these post-hoc analyses according to the reviewer's comment.

$440{ }^{3}$ In particular, detection to low contrast stimuli on a non-gamma-corrected monitor are often easier

441 than that on a gamma-corrected monitor: There might be differences in performances for the lowest

442 contrast stimuli between laboratory and online repetition conditions. Thus, according to the

443 reviewer's suggestion, we performed a two-way ANOVA on proportions of the correct responses

444 in the lowest contrast stimuli with spatial frequency $(0.5,1,2$, and $8 \mathrm{cpd})$ as a within-participant

445 factor and experimental circumstances (laboratory and online repetition) as a between-participant

446 factor. As a result, while the main effect of spatial frequency was significant $(F(3,306)=3.34, p$

$447=.02, \eta_{p}{ }^{2}=.03$ ), the main effect of experimental circumstances and interaction were not significant 
448 (experimental circumstances: $F(1,102)=0.02, p=.89, \eta_{p}{ }^{2}<.001$; interaction: $F(3,306)=0.27, p$ $\left.449=.84, \eta_{p}{ }^{2}=.003\right)$. Thus, at least, the differences in performances for the lowest contrast stimuli 450 were not found in the present study. 


\section{References}

453 Allard, R., \& Faubert, J. (2008). The noisy-bit method for digital displays: Converting a 256

454

455

456

457

458

459

460

461

462

463

464

465

466

467

468

469

470

471

472

473

474

475 luminance resolution into a continuous resolution. Behavior Research Methods, 40, 735743.

Aust, F., Diedenhofen, B., Ullrich, S., \& Musch, J. (2012). Seriousness checks are useful to improve data validity in online research. Behavior Research Methods, 45(2), 527-535. doi: $10.3758 / \mathrm{s} 13428-012-0265-2$

Bang, J. W., Shekhar, M., \& Rahnev, D. (2019). Sensory noise increases metacognitive efficiency. Journal of Experimental Psychology: General, 148, 437-452. doi: 10.1037/xge0000511

Barnhoorn, J. S., Haasnoot, E., Bocanegra, B. R., \& van Steenbergen, H. (2014). QRTEngine: An easy solution for running online reaction time experiments using Qualtrics. Behavior Research Methods, 1-12. doi: 10.3758/s13428-014-0530-7

Berinsky, A. J., Huber, G. A., \& Lenz, G. S. (2012). Evaluating online labor markets for experimental research: Amazon.com's Mechanical Turk. Political Analysis, 20, 351-368. doi: 10.1093/pan/mpr057

Berinsky, A. J., Margolis, M. F., \& Sances, M. W. (2016). Can we turn shirkers into workers? Journal of Experimental Social Psychology, 66, 20-28. doi: 10.1016/j.jesp.2015.09.010

Brady, T. F., \& Alvarez, G. A. (2011). Hierarchical encoding in visual working memory: Ensemble statistics bias memory for individual items. Psychological Science, 22, 384-392.

Brady, T. F., Shafer-Skelton, A., \& Alvarez, G. A. (2017). Global ensemble texture representations are critical to rapid scene perception. Journal of Experimental Psychology: Human Perception and Performance, 43, 1160-1176.

Brainard, D. H. (1997). The psychophysics toolbox. Spatial Vision, 10, 433-436. doi: $10.1163 / 156856897 X 00357$ 
476 Brown, H. R., Zeidman, P., Smittenaar, P., Adams, R. A., McNab, F., Rutledge, R. B., \& Dolan,

477

478

479

480

481

482

483

484

485

486

487

488

489

490

491

492 493

494 495

496

497 498

499

R. J. (2014). Crowdsourcing for cognitive science - The utility of smartphones. PLoS ONE, 9 (7), e100662. doi: 10.1371/journal.pone.0100662

Cameron, E. L., Tai, J. C., \& Carrasco, M. (2002). Covert attention affects the psychometric function of contrast sensitivity. Vision Research, 42, 949-967.

Chandler, J., Mueller, P., \& Paolacci, G. (2014). Nonnaïveté among Amazon Mechanical Turk workers: consequences and solutions for behavioral researchers. Behavior Research Methods, 46 (1), 112-130. doi: 10.3758/s13428-013-0365-7

Chaya, K., Xue, Y., Uto, Y., Yao, Q., \& Yamada, Y. (2016). Fear of eyes: Triadic relation among social anxiety, trypophobia, and discomfort for eye cluster. PeerJ, 4:e1942. doi: 10.7717/peerj. 1942

Chrabaszcz, J. S., Tidwell, J. W., \& Dougherty, M. R. (2017). Crowdsourcing prior information to improve study design and data analysis. PLOS ONE, 12: e0188246. doi: 10.1371/journal.pone.0188246

Cohen, J. (1988). Statistical power analysis for the behavioral sciences (2nd ed.). Hillsdale, NJ: Lawrence Erlbaum.

Crangle, C. E., \& Kart, J. B. (2015). A questions-based investigation of consumer mental-health information. PeerJ, 3:e867. doi: 10.7717/peerj.867

Crump, M. J. C., McDonnell, J. V., \& Gureckis, T. M. (2013). Evaluating Amazon’s Mechanical Turk as a tool for experimental behavioral research. PloS ONE, 8 (3), e57410. doi: 10.1371/journal.pone.0057410

97 de Leeuw, J. R. (2015). jsPsych: A JavaScript library for creating behavioral experiments in a web 98 browser. Behavior Research Methods, 47, 1-12.

de Leeuw, J\& Motz, B. A. Psychophysics in a Web browser? Comparing response times collected

Peer) reviewing PDF | (2019:09:40944:3:0:NEW 21 Nov 2019) 
with JavaScript and Psychophysics Toolbox in a visual search task. Behavior Research

501 Methods, 48, 1-12.

502 Garcia, D., Kappas, A., Küster, D., \& Schweitzer, F. (2016). The dynamics of emotions in online 503 interaction. Royal Society Open Science, 3:8. doi: 10.1098/rsos.160059

504 Gottlieb, S., \& Lombrozo, T. (2018). Can Science Explain the Human Mind? Intuitive Judgments 505 506 About the Limits of Science. Psychological Science, 29, 121-130. doi:

507 508

509

510

511

512

513

514

515

516

517

518

519

520

521

522

523 $10.1177 / 0956797617722609$

Holm, S. (1979). A simple sequentially rejective multiple test procedure. Scandinavian Journal of Statistics, 6, 65-70. doi: 10.2307/4615733

Hurling, R., Murray, P., Tomlin, C., Warner, A., Wilkinson, J., York, G., Linley, P. A., Dovey, H., Hogan, R. A., Maltby, J., \& So, T. T. (2017). Short Tips Delivered "in the Moment" Can Boost Positive Emotion. International Journal of Psychological Studies, 9, 88-106. doi: $10.5539 /$ ijps.v9n1p88

Lafer-Sousa, R., Hermann, K. L., \& Conway, B. R. (2015). Striking individual differences in color perception uncovered by "the dress" photograph. Current Biology, 25, R1-R2. doi: 10.1016/j.cub.2015.04.053

Lakens, D., Scheel, A. M., \& Isager, P. M. (2018). Equivalence Testing for Psychological Research: A Tutorial. Advances in Methods and Practices in Psychological Science, 1, 259-269.

Lee, T. H., Baek, J., Lu, Z. L., \& Mather, M. (2014). How arousal modulates the visual contrast sensitivity function. Emotion, 14, 978-984.

Litman, L., Robinson, J., \& Abberbock, T. (2017). TurkPrime. com: A versatile crowdsourcing data acquisition platform for the behavioral sciences. Behavior Research Methods, 49, 433-442. doi: 10.3758/s13428-016-0727-z 
524 Lu, Z\& Dosher, B. (2013). Visual Psychophysics: From Laboratory to Theory. MIT Press.

525 Majima, Y. (2017). The feasibility of a Japanese crowdsourcing service for experimental research 526 in psychology. SAGE Open, 7, 1-12.

527 Maniaci, M. R., \& Rogge, R. D. (2014). Caring about carelessness: Participant inattention and its 528 effects on research. Journal of Research in Personality, 48, 61-83. doi:

529 10.1016/j.jrp.2013.09.008

530 Miura, A., \& Kobayashi, T. (2016). Survey satisficing inflates stereotypical responses in online 531 experiment: The case of immigration study. Frontiers in Psychology, 7:1563. doi:

532 10.3389/fpsyg. 2016.01563

533 Nosek, B. A., Banaji, M., \& Greenwald, A. G. (2002). Harvesting implicit group attitudes and 534 beliefs from a demonstration web site. Group Dynamics: Theory, 6 (1), 101-115. doi:

535 $10.1037 / 1089-2699.6 .1 .101$

536

537

538

Oppenheimer, D. M., Meyvis, T., \& Davidenko, N. (2009). Instructional manipulation checks: Detecting satisficing to increase statistical power. Journal of Experimental Social Psychology, 45 (4), 867-872. doi: 10.1016/j.jesp.2009.03.009

539 Palan, S., \& Schitter, C. (2018). Prolific.ac-A subject pool for online experiments. Journal of 540 Behavioral and Experimental Finance, 17, 22-27.

541 Pechey, R., Attwood, A. S., Couturier, D. L., Munafò, M. R., Scott-Samuel, N. E., Woods, A., \& 542 Marteau, T. M. (2015). Does glass size and shape influence judgements of the volume of wine? PLOS ONE, 10: $\mathrm{e} 0144536$.

Pelli, D. G. (1997). The VideoToolbox software for visual psychophysics: Transforming numbers 545 into movies. Spatial Vision, 10, 437-442. doi: 10.1163/156856897X00366

546 Pinet, S., Zielinski, C., Mathot, S., Dufau, S., Alario, F. X., \& Longcamp, M. (2017). Measuring 547 sequences of keystrokes with jsPsych: Reliability of response times and inter-keystroke 
intervals. Behavior Research Methods, 49, 1163-1176.

549 Plant, R. R., \& Turner, G. (2009). Millisecond precision psychological research in a world of 550 commodity computers: new hardware, new problems? Behavior Research Methods, 41

551 (3), 598-614. doi: 10.3758/s13428-016-0776-3

552

553

554

555

556

557

558

559

560

561

562

563

564

565

566

567

568

569

570

571

Reimers, S., \& Stewart, N. (2015). Presentation and response timing accuracy in Adobe Flash and HTML5/JavaScript Web experiments. Behavior Research Methods, 47, 309-327. doi: 10.1016/j.chb.2015.12.049

Sasaki, K., Ihaya, K., \& Yamada, Y. (2017). Avoidance of novelty contributes to the uncanny valley. Frontiers in Psychology, 8: 1792. doi: 10.3389/fpsyg.2017.01792

Schubert, T. W., Murteira, C., Collins, E. C., \& Lopes, D. (2013). ScriptingRT: A Software Library for Collecting Response Latencies in Online Studies of Cognition. PLOS ONE, 8 (6), e67769. doi: 10.1371/journal.pone.0067769

Simcox, T., \& Fiez, J. A. (2014). Collecting response times using Amazon Mechanical Turk and Adobe Flash. Behavior Research Methods, 46, 95-111. doi: 10.3758/s13428-013-0345y

Sowden, P. T., Rose, D., \& Davies, I. R. L. (2002). Perceptual learning of luminance contrast detection: Specific for spatial frequency and retinal location but not orientation. Vision Research, 42, 1249-1258. http://doi.org/10.1016/S0042-6989(02)00019-6

Stewart, N., Chandler, J., \& Paolacci, G. (2017). Crowdsourcing Samples in Cognitive Science. Trends in Cognitive Sciences, 21, 736-748. doi: 10.1016/j.tics.2017.06.007

Szafir, D. A., Stone, M., \& Gleicher, M. (2014). Adapting color difference for design. Proceedings of Color and Imaging Conference, 2014, 228-233.

To, L., Woods, R. L., Goldstein, R. B., \& Peli, E. (2013). Psychophysical contrast calibration. Vision Research, 90, 15-24. doi: 10.1016/j.visres.2013.04.011 
572 Tran, M., Cabral, L., Patel, R., \& Cusack, R. (2017). Online recruitment and testing of infants with 573 Mechanical Turk. Journal of Experimental Child Psychology, 156, 168-178. doi:

574 10.1016/j.jecp.2016.12.003

575 Turpin, A., Lawson, D. J., \& McKendrick, A. M. (2014). PsyPad: a platform for visual 576 psychophysics on the iPad. Journal of Vision, 14 (3):16, 1-7. doi: 10.1167/14.3.16

577 Ware, C., Turton, T. L., Bujack, R., Samsel, F., Shrivastava, P., \& Rogers, D. H. (2018). Measuring 578

579 and Modeling the Feature Detection Threshold Functions of Colormaps. IEEE transactions on visualization and computer graphics. doi: 10.1109/TVCG.2018.2855742

580

581

582

Woods, K. J., Siegel, M. H., Traer, J., \& McDermott, J. H. (2017). Headphone screening to facilitate web-based auditory experiments. Attention, Perception, \& Psychophysics, 79, 2064-2072.

583 Woods, A. T., Velasco, C., Levitan, C. A., Wan, X., \& Spence, C. (2015). Conducting perception 584 research over the internet: A tutorial review. PeerJ, 3:e1058. doi: 10.7717/peerj.1058

585

Yamada, Y. (2015). Gender and age differences in visual perception of pattern randomness. 586 Science Postprint, 1 (2): e00041. doi: 10.14340/spp.2015.01A0002

587

Yu, C., Klein, S. A., \& Levi, D. M. (2004). Perceptual learning in contrast discrimination and the 588 (minimal) role of context. Journal of Vision, 4 (3), 4-14. http://doi.org/10.1167/4.3.4

589

Zwaan, R. A., Pecher, D., Paolacci, G., Bouwmeester, S., Verkoeijen, P., Dijkstra, K., \& 590 Zeelenberg, R. (2018). Participant nonnaiveté and the reproducibility of cognitive

591 psychology. Psychonomic Bulletin \& Review, 25, 1968-1972. doi: 10.3758/s13423-017-

592 $1348-y$ 
596 We would like to thank Dr. Daiichiro Kuroki for developing the program of the online experiment.

597 This research was supported by JSPS KAKENHI \#17J05236 to K.S. and \#15H05709, \#16H03079, $598 \# 16 H 01866, \# 17 H 00875, \# 18 H 04199$, and \#18K12015 to Y.Y.

599 


\section{Competing interests}

601 The authors declare no competing interests.

602 
604 Contributed to conception and design: KS, YY

605 Contributed to acquisition of data: KS

606 Contributed to analysis and interpretation of data: KS

607 Drafted and/or revised the article: KS, YY

608 Approved the submitted version for publication: KS, YY

609 
610

Data accessibility statement

611 The dataset and the $\mathrm{R}$ codes for the analyses are shown in $612 \quad \underline{\text { https://figshare.com/s/c067967c1cfd3238244b }}$

613

614

615 
616 Figure legends

617 Figure 1. Timeline of a trial in all the conditions. For enhanced visibility, we presented the stimulus

618 in $100 \%$ contrast level in this figure.

619 Figure 2. Results of the correct responses in the laboratory and online experiments.

620 Figure 3. Results of the thresholds in the laboratory and online experiments. Error bars denote 621 standard deviations.

622

623 Table legends

624 Table 1. Summary of the results in differences and equivalences between laboratory, repeated, and 625 non-repeated online conditions. Note: NHST 95\% CI = Null Hypothesis Significant Test 95\% 626 confidence interval, for cases of a significant difference between pairs; TOST $90 \% \mathrm{CI}=$ Two 627 One-Sided Test 90\% confidence interval, for cases of a (marginally) significant equivalence 628 between pairs.

629 
Figure 1

Timeline of a trial in all the conditions

For enhanced visibility, we presented the stimulus in $100 \%$ contrast level in this figure. 


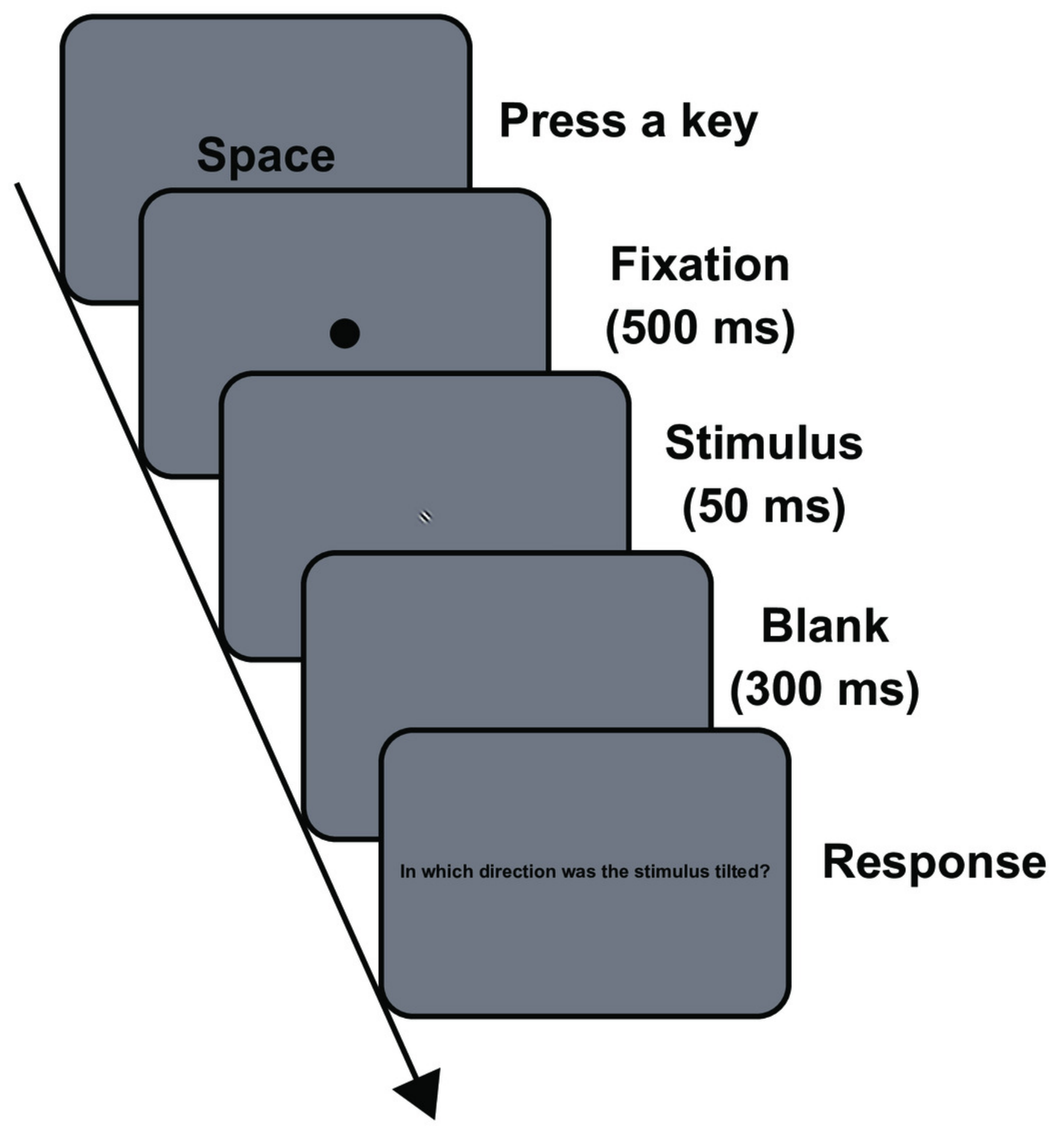


Figure 2

Microsoft Word - RevMS3rd_CrowdContrast_20191121_notracked.docx Results of the correct responses in the laboratory and online experiments.
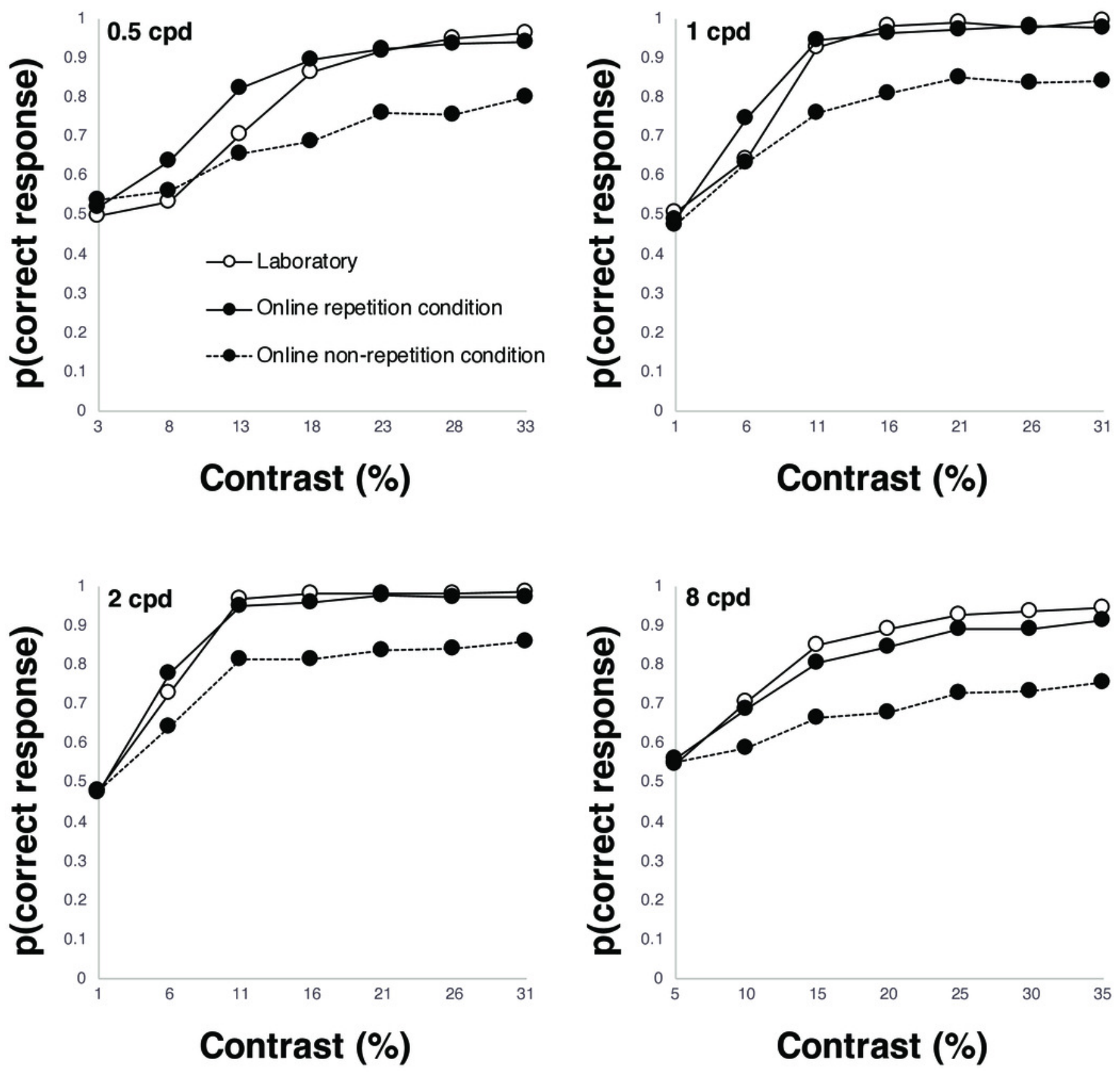
Figure 3

Results of the thresholds in the laboratory and online experiments.

Error bars denote standard deviations. 


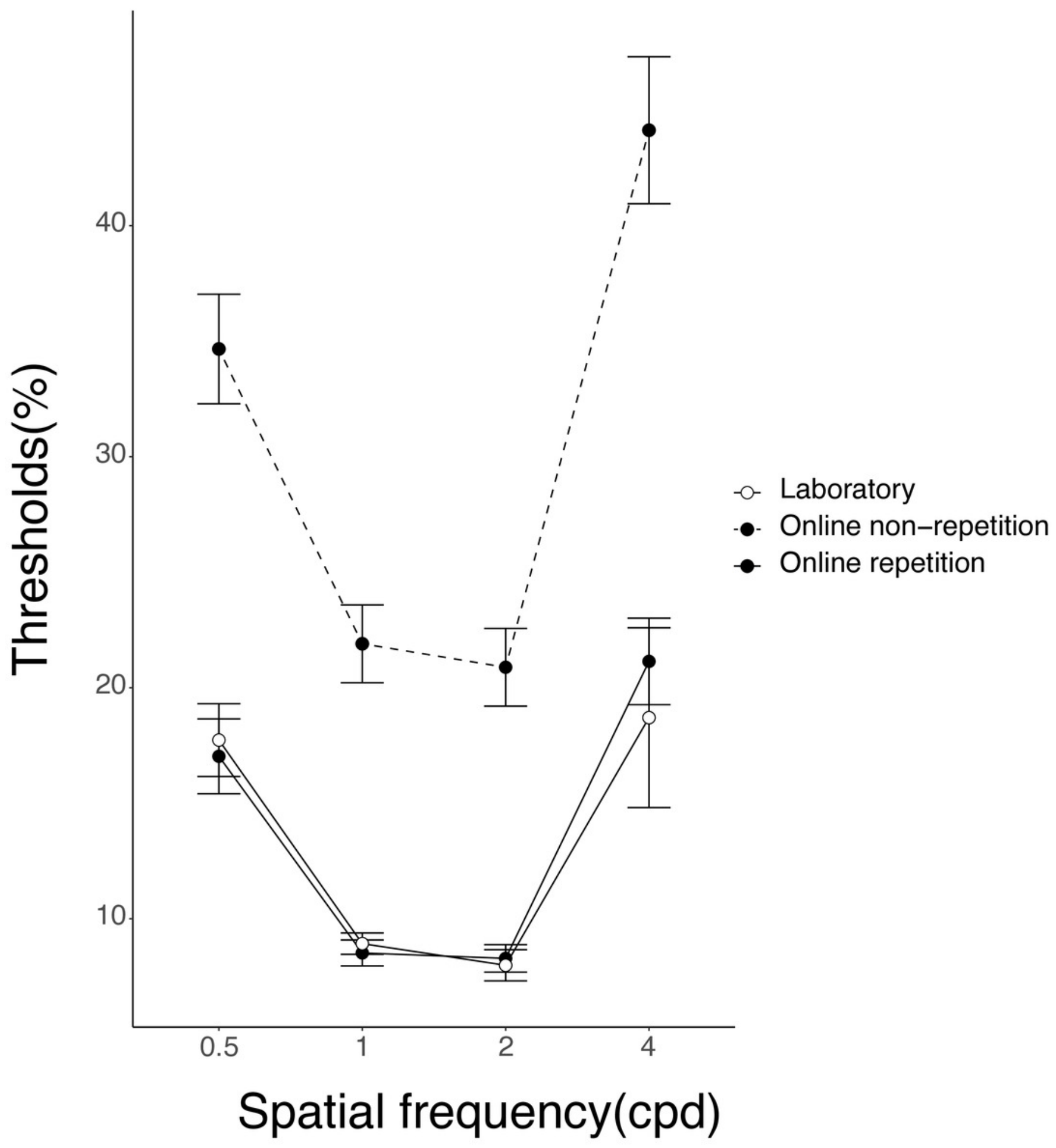




\section{Table $\mathbf{1}$ (on next page)}

Summary of the results in differences and equivalences between laboratory, repeated, and non-repeated online conditions.

NHST $95 \% \mathrm{Cl}=$ Null Hypothesis Significant Test $95 \%$ confidence interval, for cases of a significant difference between pairs; TOST $90 \% \mathrm{Cl}=$ Two One-Sided Test $90 \%$ confidence interval, for cases of a (marginally) significant equivalence between pairs. 


\begin{tabular}{|c|c|c|c|c|}
\hline cpd & & Laboratory & Online repetition & Online non-repetition \\
\hline \multirow{3}{*}{0.5} & Laboratory & & & \\
\hline & Online repetition & $\begin{array}{c}\text { Sig. Eq. } \\
\text { TOST } 90 \% \text { CI: }-3.1-4.5\end{array}$ & & \\
\hline & Online non-repetition & $\begin{array}{c}\text { Sig. Dif. } \\
\text { NHST 95\% CI: }-22.6--11.3\end{array}$ & $\begin{array}{c}\text { Sig. Dif. } \\
\text { NHST 95\% CI: }-23.3--12.0\end{array}$ & \\
\hline \multirow{3}{*}{1} & Laboratory & & \multirow[b]{3}{*}{$\begin{array}{c}\text { Sig. Dif. } \\
\text { NHST 95\% CI: }-16.9--9.9\end{array}$} & \\
\hline & Online repetition & $\begin{array}{c}\text { Sig. Eq. } \\
\text { TOST } 90 \% \text { CI: }-0.8-1.6\end{array}$ & & \\
\hline & Online non-repetition & $\begin{array}{c}\text { Sig. Dif. } \\
\text { NHST } 95 \% \text { CI: }-16.4--9.5\end{array}$ & & \\
\hline \multirow{3}{*}{2} & Laboratory & & \multirow[b]{3}{*}{$\begin{array}{c}\text { Sig. Dif. } \\
\text { NHST } 95 \% \text { CI: }-16.1--9.1\end{array}$} & \\
\hline & Online repetition & $\begin{array}{c}\text { Sig. Eq. } \\
\text { TOST } 90 \% \text { CI: }-1.8-1.2\end{array}$ & & \\
\hline & Online non-repetition & $\begin{array}{c}\text { Sig. Dif. } \\
\text { NHST 95\% CI: }-16.5--9.3\end{array}$ & & \\
\hline \multirow{3}{*}{4} & \multicolumn{2}{|l|}{ Laboratory } & & \\
\hline & Online repetition & $\begin{array}{c}\text { Marg. Sig. Eq. } \\
\text { TOST } 90 \% \text { CI: }-9.8-4.9\end{array}$ & & \\
\hline & Online non-repetition & $\begin{array}{c}\text { Sig. Dif. } \\
\text { NHST 95\% CI: }-35.5--15.4\end{array}$ & $\begin{array}{c}\text { Sig. Dif. } \\
\text { NHST } 95 \% \text { CI: }-30.3--15.7\end{array}$ & \\
\hline
\end{tabular}

